\title{
El papel de la comunicación social en la aplicación del derecho a la alimentación y la lucha contra el hambre mundial
}

institucional.us.es/ambitos/

\section{Miguel Ángel Martín López}

Universidad de Sevilla

English Version: The role of social communication in the implementation of the right to food and the fight against world hunger.

El presente escrito analiza las campañas de comunicación social para la movilización a favor del derecho a la alimentación y para la lucha contra el hambre puestas en práctica hasta la fecha. Se analizan tanto las campañas ejecutadas por las organizaciones provenientes de la sociedad civil y Ongs como las impulsadas a través de las Naciones Unidas y sus organismos especializados. Se pretende, fundamentalmente, proponer que el importante objetivo de desarrollo sostenible de acabar con el hambre para el año 2030 venga acompañado con una campaña de este corte con la más amplia participación de todas las instituciones y de la sociedad civil, diseñada para la mayor efectividad y teniendo como eje central al derecho a la
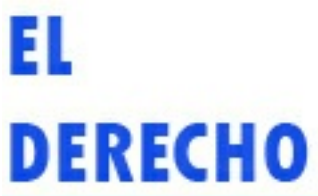
a $\mathbf{~ a ~}$

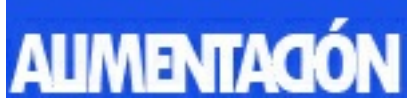
alimentación.

Palabras clave: Derecho a la Alimentación, Seguridad Alimentaria, Lucha contra el Hambre, Hambre Cero, Campaigning, Campañas de Comunicación, Objetivos de Desarrollo Sostenible de Naciones Unidas.

\begin{abstract}
The current writing analises the social communication campaigns for the mobilization in favour of the right to the food and the fight against hunger implemented to date. Both the campaigns execute by organizations from civil society and NGOs and those promoted through the United Nations and its specialized organism are analyzed. It is fundamentally intended to propose that the important goal of sustainable development to end hunger by the year 2030 be accompanied by a campaign of this cort with the broadest participation of all institutions and civil society, designed for the most effective and having as central axis the right to food.
\end{abstract}

Keywords: Right to Food, Food Security, Fight against Hunger, Zero Hunger, Campaigning, Communication Campaigns, United Nations Sustainable Development Goals.

\section{Introducción}

Un aspecto que no suele ser tomado en cuenta en la defensa y promoción del derecho a la alimentación es la comunicación social. No cabe duda que el recurso de los medios de comunicación es de sumo interés para involucrar a la sociedad civil en esta labor, facilitando el cambio y las transformaciones sociales. La adopción de posturas favorables para la realización de este derecho y, por tanto, para la erradicación del hambre en el mundo tiene que ser impulsado. Habría que explotar este potencial transformador, ya que no solo a través del derecho y la adopción de normas se consigue el cumplimiento de los objetivos pretendidos.

De manera indirecta, podemos entender que las Directrices voluntarias de la FAO para la realización progresiva del derecho a la alimentación reconoce la importancia de esta labor, en concreto, a través de la directriz undécima, dedicada a la educación y sensibilización.

Expresamente, esta regla indica que los Estados deberían incrementar el conocimiento de las presentes directrices por parte de sus ciudadanos y alienta a promover la concienciación de la ciudadanía de los derechos 
humanos. También hay referencias, aunque generales y poco concretas, a que los Estados formen a sus funcionarios, a que establezcan programas escolares, inversiones en recursos humanos que incluyan educación sobre agricultura y medio ambiente en la enseñanza básica, secundaria y superior.

A la hora de prever medidas de participación de la sociedad civil en la aplicación de las directrices es más cauta, la redacción del texto es más cauta. Así, se indica en el último parágrafo de la referida undécima directriz que los Estados tal vez deseen dotar a la sociedad civil de los medios necesarios para que participe en la aplicación de las directrices, por ejemplo, mediante la creación de capacidades.

Como vemos, la redacción de esta regla utiliza la expresión "tal vez", de la cual no se denota una amplia intención de compromiso. Se deja a la plena voluntad de los Estados.

\section{Las Campañas de promoción y defensa del derecho a la alimentación por parte de las organizaciones de la sociedad civil}

Sin lugar a dudas, el movimiento ciudadano y las organizaciones de la sociedad civil han sido activas en esta temática y vienen llevando a cabo campañas de defensa del derecho a la alimentación, bien articuladas y con trayectoria. Probablemente, la más exitosa y de impacto a nivel internacional es la que se viene llevando en la India con el título Right to Food Campaing (véase www.righttofoodcampaig.in). Esta campaña nació en 2001 y surge de un movimiento informal desde debajo de particulares y organizaciones, que empezó recogiendo apoyo para participar y estar presentes en un asunto sobre el tema ante el Tribunal Supremo del país y que ya se conforma como todo un movimiento nacional.

Cuenta con buen número de voluntarios, buenos mecanismos de difusión y una clara definición de las herramientas de acción, intentando influir en la acción legal y normativa (litigando ante los tribunales, vigilando el cumplimiento de las sentencias del tribunal supremo, visitas de campo, etc.). Realizan también materiales de difusión y de investigación, los llamados materiales de campaña, que no se quedan en consideraciones generales, sino que abordan cuestiones prácticas de acción sobre temática concreta (nutrición a niños menores de seis años, sistemas de distribución pública, derechos en maternidad, ámbito rural, etc.). Es, fundamentalmente, un trabajo de advocacy bien estructurado, lo que empieza ya a estructurarse bien en la escena internacional, como puede verse en trabajos de considerable interés y profundidad existentes en la bibliografía internacional (Pride, Richard 2003, Mundy, Karen and Lynn, Murphy 2001).

Pueden encontrarse otras campañas y, en este sentido, destaca una que está teniendo lugar en nuestro país. Se trata de Derecho a la Alimentación urgente (https://derechoalimentacion.org/). Esta campaña de sensibilización está promovida por varias organizaciones no gubernamentales (Acción contra el Hambre, Ayuda en Acción, Cáritas, ONGAWA), siendo coordinada por Prosalus. La campaña ha sido muy activa y tiene un buen número de materiales y guías prácticas de utilización para la puesta en práctica del derecho a la alimentación.

También puede mencionarse a nivel internacional, el lanzamiento de una red internacional en 2013, auspiciada principalmente por la organización especializada en la materia FIAN denominada Global Network for the Right to Food and Nutrition. Esta red cuenta también con una página web, donde es posible consultar todo el programa y labor que realizan : http://www.righttofoodandnutrition.org/. La red está abierta a la participación de los movimientos sociales y las organizaciones de la sociedad civil de interés público, incluso las personas particulares pueden unirse a la misma cumplimiento unos requisitos. Es una plataforma de unificación de un buen número de las organizaciones y plataformas más activas en la defensa de este derecho humano. Es, en esencia, un consorcio de organizaciones. Recoge noticias y documentos y pretende dar una llamada de opinión a la opinión pública mundial y a la acción de los gobiernos. No actúa, sin embargo, a través de los medios de comunicación, intentando influir desde allí. 


\section{Desarrollo Sostenible para el año 2030}

Como estamos viendo en todos estos casos, son organizaciones vinculadas a la sociedad civil sin respaldo gubernamental o de las organizaciones internacionales, como las Naciones Unidas. Cabría preguntarse si éstas vienen impulsando alguna campaña de este corte a favor del derecho a la alimentación.

En este sentido, hay que indicar que con dedicación expresa al derecho a la alimentación no se puede encontrar. Ahora bien, desde la organización de Naciones Unidas para la agricultura y la alimentación (FAO) se han desarrollado algunas campañas en los últimos años contra el hambre.

Así, es del máximo interés recordar la campaña que llevó a cabo dicho organismo especializado a partir de mayo de 2010, poco después de la crisis alimentaria mundial que provocó una drástica subida del precio de los alimentos básicos y, por tanto, del número total de personas en situación de hambre e inseguridad alimentaria en la escena mundial. Se había llegado a superar la cifra de mil millones de personas (one billion people, en la expresión de lengua inglesa). La perspectiva en aquel momento también mostraba que no iba a ser posible el cumplimiento del objetivo del milenio relacionado con el hambre.

La campaña fue, desde el punto de vista comunicativo, muy directa con un mensaje contundente y movilizador. Buscó también la búsqueda de aliados, empezando por el conocido y prestigioso actor Jeremy Irons y tuvo una considerable repercusión en los medios de comunicación. El actor británico, emulando una conocida escena de un presentador de televisión en la película Network de 1976, manifiesta su enfado contra la insostenible situación del hambre en la comunidad internacional : " I'm mad as hell ». A partir de ahí, se hace un llamamiento a la sociedad civil para que la gente demuestre su enfado ante dicha cifra del billón inglés de personas hambientas firmando una petición dirigida a los gobiernos a través de internet (www.1billionhungry.org. La página ya no está operativa en la actualidad, apareciendo valoraciones de hoteles).

La campaña hacía un amplio uso de las redes sociales (facebook, twitter), youtube y se completaba con eventos en diferentes ciudades del planeta y el apoyo de otras personas conocidas. La propia FAO señalaba que se utilizaba un tipo de letra y un lenguaje atrevido para atraer la atención de la gente y para recalcar que la situación en esta cuestión ha llegado al límite. Se creaba también un símbolo, como era el silbato amarillo, representativo y muestra del enfado contra el hambre mundial.

El propósito era muy concreto y específico, lo cual es positivo al concretar el objetivo y resultado a conseguir. No obstante, la campaña fue efímera, sin ánimo de tener mayor calado, quedando agotada. Además, no llegaba en ningún momento a mencionar al derecho a la alimentación.

Tras esta campaña, podemos encontrar el impulso de otra de las pretensiones ambiciosas en el marco de la FAO y de las Naciones Unidas dedicada igualmente a la lucha contra el hambre y que se ha venido promoviendo desde el año 2012 por el anterior secretario general, Ban Ki Moon. El nombre utilizado para ello es « Reto del Hambre Cero » (zero hunger). Utiliza un nombre que hace recordar al programa auspiciado por el gobierno de Brasil, en el mandato de Luis Ignacio Da Silva y que tan buenos resultados ha dado en esta lucha.

Ahora bien, las pretensiones son mucho más modestas. La campaña tiene objetivos más reducidos e incluso, vista la acción llevada a cabo en estos años, resultados muy limitados.

El Reto del Hambre Cero pretendía movlilizar esfuerzos de agentes diversos de la sociedad internacional para lograr acabar con el hambre, Se trata de asumir una breve declaración de intenciones : « trabajar activamente para acabar con el hambre, eliminar todas las formas de malnutrición... la construcción de sistemas alimentarios inclusivos y sostenibles... y apoyar la presente iniciativa ». Naturalmente, un compromiso laudable, aunque no puede pasar inadvertido que este reto no menciona ni se vincula al derecho a la alimentación.

Se quiere denominar también movimiento mundial para el hambre cero, contando con su logotipo y materiales promocionales e incluso con una amplia página web : www.un.org.zerohunger. Quiere conseguir un apoyo global, abriéndose a Ongs, empresas, sociedad civil, aunque, a la fecha, el predicamento y alcance de este movimiento no es muy amplio y su eco es reducido. Si observamos la información aportada en dicha página web, encotramos una cifra de cuarenta y nueve participantes en esa categoría de miembros de la sociedad civil 
y empresas. Podemos bien pensar que es una cifra muy modesta. Sorprende además que están las más grandes multinacionales del sector agroalimentario, Cargill, Univelever, por ejemplo, o la asociación mundial de productores de fertilizantes.

La actual adopción del compromiso internacional de acabar con el hambre para el año 2030, el segundo de los objetivos de desarrollo sostenible de las Naciones Unidas debiera ser un revulsivo para darle un mayor valor a la comunicación social y a esta campaña en particular para buscar el compromiso activo de la ciudadanía en general y motivar una participación eficaz y eficiente para acabar realmente con el hambre en dicha fecha de 2030.

Este objetivo es ambicioso y, desde luego, no va a poder ser cumplido si no se es igualmente ambicioso en los medios dispuestos para ello. La FAO cifra para ello una cantidad de dinero de 267.000 millones de dólares (http://www.abc.es/natural-desarrollorural/20150713/abci-hambre-cero-201507131150.html), pero además de la inversión, sería necesario fortalecer el derecho a la alimentación, conseguir la voluntad política real de los gobiernos y la implicación y empuje de la sociedad civil mundial en este propósito.

En su consecuencia, creemos que este llamado Reto del Hambre Cero debe incluir al derecho a la alimentación. No es un mensaje coherente que haya esfuerzos en la lucha contra el hambre diversos y no conectados entre sí. Debe ser además la oportunidad para dar entrada y efectividad al referido papel de la comunicación y participación social.

Es una propuesta lógica para ver los progresos, evaluar los avances y hacer un seguimiento del reto. Creemos que el modelo señalado de la campaña que se está llevando a cabo en la India es de gran interés al respecto. No se queda en un mesaje general y abstracto sino que se desagrega en objetivos bien diseñados y concretos que se van viendo incluso exigiendo judicialmente.

Aunque aún de alcance limitado, si es interesante señalar que se Naciones Unidas está empezando a diseñar una iniciativa siguiendo esta lógica. Este es el caso de la iniciativa mundial sobre la reducción de la pérdida y desperdicio de la alimentación, piensa-alimenta.ahorra y que puede verse en la página web www.thinkeatsave.org. Se incluye además en las páginas del Reto de Hambre Cero. La iniciativa fue lanzada oficialmente el pasado 30 de noviembre de 2016 en el Reffetorio Gastromotiva en Rio de Janeiro y reconoce que los poderes públicos no pueden por sí mismos reducir estas pérdidas y desperdicios, de ahí que se busque ampliar la acción creando una gran alianza. Se invita a participar a todas las compañías del sector privado, integrantes en la cadena de suministros o que provean servicios a la misma, y a las organizaciones de la sociedad civil de todo el mundo. También se indica expresamente que pueden unirse a la red las universidades, departamentos gubernamentales, organizaciones no gubernamentales de cooperación o asociaciones de los países en desarrollo.

En materia de nutrición, una cuestión tan esencial para el derecho a la alimentación, sobre todo para los niños y niñas, también debiera establecerse una iniciativa similar concreta y de amplia motivación y participación. Ello se deduce de la recomendación vigesimoprimera del marco para la acción aprobado en la reciente conferencia mundial para la nutrición y que tuvo lugar en Roma en noviembre de 2014. Expresamente, se dice que hay que emprender campañas de concienciación social y programas de comunicación sobre cambios en el estilo de vida que promuevan la actividad física, la diversificación dietética y el consumo de alimentos ricos en micronutrientes tales como frutas y hortalizas, con inclusión de alimentos locales tradicionales y tomando en cuenta las consideraciones de índole cultural, así como mejoras en la nutrición maternoinfantil, prácticas de cuidado apropiadas y la lactancia materna y alimentación complementaria adecuadas, orientadas y adaptadas a los diversos públicos y grupos de interesados dentro del sistema alimentario (EB136/8 15 Anexo 2, marco de acción, Segunda Conferencia Internacional sobre Nutrición (CIN2), Roma 19-21 noviembre 2014, FAO y Organización Mundial para la Salud).

Incluso también debiera incluirse al derecho a la alimentación en la acción de comunicación en materia de cambio climático. Bien ha quedado en este último campo patente la necesidad de usar la comunicación social y se está previendo su inclusión tanto en los planes internacionales como en la legislación nacional e incluso andaluza. En este último caso se prevé un Programa de Comunicación del Plan Andaluz de Acción por el Clima, 
como hace el artículo 25 del recientemente aprobado anteproyecto de la ley andaluza contra el cambio climático. El respetado diario británico The Guardian es el que más está poniendo de manifiesto y alertando a la opinión pública sobre esta peligrosa connivencia entre ambos fenómenos. En su nota de 16 de octubre de 2016 ya alertó que, para dicho año 2030, los efectos del cambio climático pueden llevar a la situación de hambre y pobreza de 122 millones de personas más, provenientes sobre todo del pequeño campesinado y del mundo rural de los países en desarrollo (https://www.theguardian.com/global-development/2016/oct/17/climate-changecould-drive-122m-more-people-into-extreme-poverty-by-2030-un-united-nations-report).

Otras notas anteriores también iban en un mismo sentido y, sobre todo, el pasado 26 de mayo del presente año 2017 publicaba una extensa entrevista con el saliente presidente Obama que recalcaba considerablemente estas ideas del vínculo cambio climático-seguridad alimentaria. Muestra la necesidad de actuar en esta dirección, ante un ámbito que no se ha dado a conocer. En sus palabras, "Food has not been the focus of climate change discussions as much as it should have been. Part of the problem is that we haven't publicised the impact of food production on greenhouse gas emissions ». También sigue diciendo que " so part of the problem that we need to address is just lack of knowledge in the general public. Keep in mind how long it took to educate people around climate change, and we still have a lot of work to do » (https://www.theguardian.com/global-development/2017/may/26/barack-obama-food-climate-change).

En este sentido, hay que decir y reconocer que algunas organizaciones no gubernamentales y de la sociedad civil han venido llevando a cabo algunas campañas para concienciar del impacto de la producción de alimentos en el calentamiento global. Sobre todo, las mismas han versado alrededor del concepto de " food miles », traducido al español como " alimentos kilométricos ». Amigos de la Tierra, en nuestro país, tiene vigente una campaña con dicho nombre y que puede verse en la página web www.alimentoskilometricos.org.

El concepto de "Food Miles " surgió ya incluso en la década de los noventa por obra del profesor londinense Tim Lang, explicitando la larga distancia que ha de recorrer mucha de la comida hasta llegar a ser consumida en nuestra mesa. El prestigioso Times ya destacó en 2006 que la comida que se iba a servir en cada cena de la próxima navidad tenía un recorrido, de media, de 84.000 millas de vuelo. El revuelo fue considerable y ha sido una cuestión muy debatida, ya que las propias compañias y empresas alimentarias y la asociación de transportistas tienen sus propias campañas e información para contrarrestar esta idea.

Ciertamente, la cuestión presenta una complejidad considerable. Los alimentos suelen llegar más por tráfico marítimo que por aéreo. También se ha contrargumentado que producir algunos cultivos en tierras menos adecuadas, genera más emisiones de $\mathrm{CO} 2$ que el coste de su transporte. Se pone incluso el ejemplo del cultivo de la fresa en España e Inglaterra. Además, algunas investigaciones auspiciadas por las compañias destacan incluso que se consume más combustible en el traslado de los particulares en sus vehículos a los centros comerciales para hacer la compra de alimentos que por el coste del transporte del alimento en sí.

La cuestión presenta una complejidad que debe requerir la creación de consensos para abordar con profundidad la influencia real de los modos de producción agrícolas actuales y también de distribución en el cambio climático y, por tanto, a la larga también en la seguridad alimentaria mundial y en el derecho a la alimentación.

Ello debe entrar en la campaña mundial y en el reto del Hambre Cero para el 2030, generándose un debate profundo y una toma de decisiones tajante y efectiva por parte de lo poderes públicos.

Hasta la fecha, si es destacable la campaña la lanzada desde el ámbito local por parte de los alcaldes en el llamado Pacto de Milán por una política alimentaria urbana (http://www.milanurbanfoodpolicypact.org/). Este pacto tiene una visión holística, de conjunto, a fin de desarrollar sistemas alimentarios sostenibles, inclusivos, resilientes, seguros y diversificados, para asegurar comida sana y accesible a todos en un marco de acción basado en los derechos, con el fin de reducir los desperdicios de alimentos y preservar la biodiversidad y, al mismo tiempo, mitigar y adaptarse a los efectos de los cambios climáticos.

Cambiando de tema, podemos concluir que la importancia de los medios de comunicación, por tanto, es esencial. Si no fuera por este medio, los informes científicos y técnicos que sirven de base a tales predicciones no tendrían difusión alguna más allá de algunos entendidos en el tema. 
El papel de la comunicación y participación social en una cuestión tan esencial como el cumplimiento del derecho a la alimentación, sobre todo en su aspecto fundamental de estar libre del hambre es esencial y debe tomarse ello en cuenta para conseguir sensiblilizar y concienciar a la población y autoridades del cumplimiento de las obligaciones que debe emanarse de este derecho. Debe servir también para facilitar la comprensión, formación y difusión del contenido de estas obligaciones. Es por ello oportuno también que estas campañas incluyan también a las instancias judiciales, tribunales, jueces y colegios de abogados, como públicos objetivos.

Vivero Pol y Andrew Mc Millan también han abogado por incluir una gran campaña en la labor de lucha contra el hambre y como acompañamiento a los tratados internacionales y textos jurídicos que deben erradicarla. En sus palabras textuales, " a campaign has to be designed to rally people across the world around this goal and engage them in the processes that will lead to its achievement. The campaign would raise public awareness and undestanding of the hunger problem and of solutions, engage policy-makers and bring an increasingly large numbrer of institutions into the consensus " (Vivero Pol, J. L. and MC Millan, Andrew, The governance of hunger, 2011, p. 79).

La formación, incluso la reflexión e investigación, debe también tener un papel preeminente en esta labor. Así, las universidades pueden catalizar esta labor en este sentido. La experiencia del Observatorio para el derecho a la alimentación en América Latina y el Caribe (http://www.oda-alc.org/) es relevante en este fin y hay que destacar los esfuerzos por la FAO para extenderlos a otros ámbitos

Pero incluso se debe profundizar más y establecer mecanismos para que instancias de la sociedad civil se encarguen de supervisar, hacer seguimiento y reinvidicar el cumplimiento del derecho a la alimentación (véase Fox, J. Veertically integrated, p. 616-627). Ha de tener incluso potestad para denunciar los incumplimientos, movilizando a la actuación pública. Ser, en definitiva, promotores de la exigibilidad de este derecho y contribuir a la vigilancia de su cumplimiento (Martin Wolpold-Bosien, La Defensa p. 201). La experiencia de la India, antes reseñada, es una buena práctica al respecto.

Estamos ante un ámbito novedoso, pero que puede permitir desarrollar. Una de las escasas investigaciones sobre el papel de la comunicación en el ámbito de la seguridad alimentaria señala muchos instrumentos y herramientas que pueden ser ensayados y utilizados en esta labor. En concreto, señalan que " white Papers, policy briefs, exhibits, community meetings, and coalitions emerge as solutions developed through our partnerships that focus on addressing specific problems related to food insecurity by fostering spaces for listening to the voices of the food insecure" (M Dutta et. al.Voices of Hunger, p. 159).

\section{Conclusiones}

En conclusión, estimamos que para que el actual compromiso internacional del reto contra el hambe, segundo objetivo de desarrollo sostenible para el año 2030 , se haga una realidad real es necesario dar la mayor eficacia al derecho a la alimentación. Todos los conceptos utilizados deben converger y ser utilizados de manera unitaria. A veces se habla de lucha contra el hambre, otras de seguridad alimentaria y otras de derecho a la alimentación. El mensaje se dispersa y, evidentemente, el contenido no es el mismo en uno y otro caso.

Hay que unificar el mensaje en una sola campaña que aglutine todos los esfuerzos de Naciones Unidas (y, sobre todo, la FAO) en estos ámbitos.

A partir de esta campaña unitaria, ya se podría desglosar el trabajo en los diversos ámbitos (desperdicio de alimentos, nutrición, cambio climático, etc), guardando en todo caso la coherencia entre los mensajes. Ello debe hacerlo de manera real y creíble y debe incluir inexorablemente al derecho a la alimentación

La campaña mundial de Naciones Unidas debiera establecer, en primer lugar, una alianza con las autoridades gubernamentales, a nivel central pero también con las administraciones de ámbito local, que permiten una mayor participación de las diferentes instancias de la sociedad civil. Deben preverse mecanismos de supervisión del cumplimiento del derecho a la alimentación y del fomento de la lucha contra el hambre. Hay que 
involucrar a todas las instancias de la sociedad civil. Se puede buscar la complicidad de los diversos estamentos mediante la firma de declaraciones y compromisos de apoyo y acción, plasmadas en acciones concretas y no abstractas, fruto del consenso entre todos los actores. Debe igualmente mantenerse una acción prolongada y sostenida en el tiempo y no meramente acciones puntuales o que se agoten. Probablemente, en este sentido, sea necesaria la creación de instituciones que perduren (comisiones de seguimiento nacionales y comisiones locales).

\section{Referencias bibliográficas}

Fox, Johnathan A (2001). Vertically Integrated Policy Monitoring: A Tool for Civil Society Policy Advocacy Nonprofit and Voluntary Sector Quarterly 30(3):616-627 · September 2001

Martin Wolpold-Bosien (2008). «La Defensa del Derecho a la Alimentación y el papel de la sociedad civil », Derecho a la Alimentación y Soberanía Alimentaria , UCO ; Córdoba, págs. 201 y ss

M Dutta, Christina Jones, Abigail Borron, Agaptus Anaele, Haijuan Gao, Sirisha Kandukuri, « Voices of Hunger: A culture-centered approach to addressing food insecurity », Journal of Communication 63 (1), 159-180

Mundy, Karen and Lynn, Murphy (2001). "Transnational Advocacy, Global Civil Society? Emerging Evidence from the Field of Education," Comparative Education Review 45, no. 1 (February 2001): 85-126.

Pride, Richard (2003). « Transnational civil society and advocacy in world politics », World Politics 55 (July), 579-606

Vivero Pol, José Luis, Mac Millan, Andrew (2011). « The governance of hunger. Innovative proposals to make the right to be free from hunger a reality », New Challenges to the Right to Food, Huygens, Barcelona.

- Recibido: 12 de junio de 2017

- Aceptado: 20 de junio de 2017

Ámbitos. Revista Internacional de Comunicación, n.37, edición de verano, 2017. 